\title{
MEDIÇÃO REMOTA DE PARÂMETROS ELÉTRICOS USANDO IOT BASEADA NO MICROCONTROLADOR ESP32
}

\author{
Amorim Jr, J. P. ${ }^{1^{*}}$; Ribeiro, C. M. ${ }^{2}$; Colistete Jr, R. ${ }^{3}$ \\ ${ }^{1}$ Bacharel em Ciência da Computação, Universidade Federal do Espírito Santo, Alegre, ES, Brasil. \\ 2,3 Departamento de Química e Física, Universidade Federal do Espírito Santo, Alegre, ES, Brasil. \\ ${ }^{1}$ josep_jr@outlook.com; ${ }^{2}$ cm_ribeiro@terra.com.br; ${ }^{3}$ roberto.colistete@ufes.br
}

\begin{abstract}
Resumo
A Internet das Coisas (IoT) traduz a ideia de objetos conectados à Internet e entre si, através de uma rede, munidos de sensores, controladores, circuitos eletrônicos e softwares, capazes de coletar, processar e trocar dados e informações. Existem diversas aplicações onde a loT é usada para dar flexibilidade aos sistemas criando assim uma estrutura distribuída, autônoma e cooperativa. Este artigo descreve a aplicação do conceito de loT no desenvolvimento de um sistema capaz de monitorar e controlar, em tempo real, dispositivos elétricos conectados a uma ilha de medição de grandezas elétricas. Nesse sentido a ilha de medição conta, essencialmente, com um circuito de condicionamento de sinais e um microcontrolador ESP32. Na ilha são realizados cálculos de grandezas elétricas de interesse do usuário, tais como: potência média, fator de potência, valor eficaz de tensão e valor eficaz de corrente. Para a realização de testes e comparação de dados utilizou-se uma fonte ajustável de tensão e cargas resistivas de diferentes potências e os resultados foram comparados com aqueles obtidos por um wattímetro convencional. O sistema desenvolvido pode ter suas funcionalidades ampliadas de tal forma que a ilha de medição passe a controlar não somente um, mas vários dispositivos simultaneamente. Também pretende-se que um microcomputador Raspberry Pi monitore não somente uma, mas várias ilhas, tornando esse sistema a base de uma casa inteligente.
\end{abstract}

\begin{abstract}
The Internet of Things (IoT) translates the idea of objects connected to the Internet and to each other, through a network, equipped with sensors, controllers, electronic circuits and software, capable of collecting, processing and exchanging data and information. There are several applications where loT is used to bring flexibility to the systems, thus creating a distributed, autonomous and cooperative structure. This paper describes the application of the loT concept in the development of a system capable of monitoring and controlling, in real time, electrical devices connected to an island of measurement of electrical quantities. In this sense, the measuring island relies essentially on a signal conditioning circuit and an ESP32 microcontroller. On the island, calculations are carried out, such as average power, power factor, RMS voltage value and RMS current value. For realization of test and comparison of data, used one voltage source adjustable and resistive load of different power and results were compared to a conventional wattmeter. The created system can have its functionality enlarged in such a way that the island does not control not only one, but several devices simultaneously. It is also intended that a Raspberry Pi microcomputer monitor not only one but also several islands, making this system the basis of a smart home.
\end{abstract}

Keywords (Palavras chaves): Internet das Coisas, llha de Medição, Grandezas Elétricas, Microcontrolador, ESP32. 


\section{Introdução}

A Internet das Coisas (Internet of Things ou loT) surgiu da necessidade de conectar "coisas" (objetos) do dia a dia à Internet e veio para facilitar a vida das pessoas. Como novo conceito de uso da Internet, ela passa a conectar não só computadores, como também objetos do dia a dia (MATTERN; FLOERKEMEIER, 2010). É um assunto que vem se difundindo em todo o mundo e é a base para se criar projetos de soluções em diversas áreas como planejamento urbano, produção agrícola, grandes produções industriais, logística, preservação do meio ambiente etc.

O cientista da computação Mark Weiser, criador do conceito Computação Ubíqua, defendia o oposto da Realidade Virtual em que as pessoas são colocadas dentro do mundo gerado pelo computador. $\mathrm{Na}$ Computação Ubíqua, é o computador que se integra a vida das pessoas de modo que elas não percebam, mas o utilizem (WEISER, 1993). Segundo Weiser (1999, p. 3), "As tecnologias mais profundas e duradouras são aquelas que desaparecem. Elas dissipam-se nas coisas do dia-a-dia até tornarem-se indistinguíveis". Diminuindo cada vez mais com a evolução da tecnologia, os computadores passaram a se tornar imperceptíveis.

A IoT conceitua a ideia de objetos conectados à Internet e entre si, através da rede, munido de sensores, controladores, circuitos eletrônicos e softwares, capazes de coletar, processar e trocar dados e informações. Existem diversas aplicações onde a loT é usada para o fortalecimento de sistemas, criando assim um grande sistema distribuído, autônomo e cooperativo. Essas redes permitem verificar uma variedade de fenômenos que são descritos por grandezas físicas. Grandezas físicas podem ser organizadas de forma a refletir grandezas "abstratas" como captura de faces, íris, digitais, lugares visitados e registrados, localização de veículos em uma estrada e até pessoas em uma multidão (AQUINO, 2015). Um objeto no qual se aplica o conceito de loT pode ser qualificado como inteligente, devido à característica de poder se comunicar com outros objetos e de poder fazer isso de forma autônoma. De acordo com o site Gartner (EGHAM, 2017), a previsão é que a partir de 2020 mais de 20,4 bilhões de objetos inteligentes estarão conectados à Internet.

O uso de serviços em geral, voltados a loT, juntamente com o conceito de automação, seja ela industrial ou residencial, é capaz de entregar ao usuário deste conjunto de serviços e aplicações algumas vantagens como: maior conforto devido ao uso de microcontroladores; melhor usabilidade, podendo ser monitorado e controlado a distância; aumento de produtividade, quando se troca aplicações manuais por aplicações automáticas; maior controle de qualidade, usando variáveis programadas podendo atingir melhores resultados e melhor custo-benefício graças aos baixos custos dos microcontroladores e microcomputadores, alcançando com todas essas vantagens uma gama de possibilidades práticas e econômicas.

\section{Materiais e Métodos}

Esse projeto engloba o desenvolvimento de uma estrutura voltada para medições de grandezas elétricas, baseado principalmente no microcontrolador ESP32.

\subsection{Hardware}

A ilha de medição de grandezas elétricas é o hardware principal tendo sido projetado e montado especificamente para o projeto. Nesta seção são descritos os componentes básicos e estratégias adotadas.

\subsubsection{Circuito de Condicionamento de Sinais}

De forma resumida, os sinais elétricos de interesse são os valores instantâneos de corrente e tensão. A partir destes valores é possível calcular indiretamente as diversas outras grandezas pretendidas. Pode-se caracterizar tensão e corrente típicas de um eletrodoméstico conectado à rede elétrica da seguinte forma: são funções periódicas, alternadas e com frequência de $60 \mathrm{~Hz}$, tensão nominal de 127 ou 220 Volts (valor eficaz) e corrente associada à potência do equipamento utilizado. Para este projeto, optou-se por otimizar o circuito de condicionamento de sinais para a tensão de 127 Volts, o que implica, para uma função senoidal, uma amplitude de 180 Volts (ou seja, um sinal que pode variar entre -180 e +180 volts). Para a corrente, optou-se por otimizar o circuito para amplitude de até 10 Ampères, compatível com uma ampla gama de eletrodomésticos. Portanto, foi necessário um circuito de condicionamento de sinais capaz de compatibilizar estas grandezas com as características de entrada do ADC (Conversor Analógico Digital) do microcontrolador.

Para a tensão foi necessário implementar um divisor que compatibilizasse uma faixa de 360 Volts pico-apico, deixando alguma margem de tolerância para sobretensão, com uma faixa de tensão permitida na entrada do canal ADC de 0 a 3,3 Volts. Mas simplesmente reduzir a amplitude do sinal não é suficiente, pois o canal ADC não aceita tensão negativa. Daí a necessidade de se introduzir um off-set, 
sobrepondo o sinal alternado a um valor DC igual à metade do valor máximo permitido na entrada do canal $\operatorname{ADC}(3,3$ Volts $/ 2=1,65$ volts $)$.

Raciocínio equivalente foi usado para a corrente com a diferença que, neste caso, o circuito precisa ainda converter corrente para tensão, o que foi conseguido por intermédio de um resistor de valor conhecido (shunt). Dado que a tensão em um resistor é proporcional à corrente que passa pelo mesmo, então ele pode ser usado como sensor de corrente, bastando que sua resistência seja conhecida.

O circuito de condicionamento de sinais é baseado no amplificador operacional LM324 ${ }^{1}$ e permite ajuste do ganho e do off-set. Os ganhos dos amplificadores são aplicados de forma inversa no software do microcontrolador, permitindo a recuperação dos valores reais das grandezas medidas, corrente e tensão.

\subsubsection{Microcontrolador Wemos Lolin ESP32}

O microcontrolador Wemos Lolin ESP32 ${ }^{2}$ é o dispositivo responsável por coordenar a ilha de medição de grandezas elétricas. Ele tem a função de ligar e desligar quaisquer dispositivos elétricos que estiverem conectados à ilha através de relés; ler e interpretar os dados de tensão e corrente que estiverem passando no circuito quando algum dispositivo elétrico estiver em funcionamento; realizar os cálculos de potência necessários a partir dos dados lidos; e, então, gerar as informações de interesse do usuário. O ESP32 possui canais ADC com precisão 12 bits $\left(2^{12}\right.$ bits), podendo representar valores entre 0 e 4095, e sua capacidade de processamento pode chegar a 240Mhz. Após ler e interpretar os dados de tensão e corrente na saída do circuito de condicionamento de sinais, o ESP32 processa esses dados gerando informações compreendidas pelo usuário. O usuário tem acesso a essas informações através de algum servidor web criado no próprio ESP32, podendo também ser acessado via conexão local, ou enviado para um microcomputador.

\subsection{Software}

O software foi implementado para rodar no microcontrolador ESP32 que fica acoplado à ilha de medição. A programação do software é feita usando a

\begin{tabular}{lllll}
\hline 1 & Especificação do dispositivo & disponível & em: \\
<http://www.alldatasheet.com/datasheet- & & \\
pdf/pdf/17871/PHILIPS/LM324.html>. & & \\
2 & Especificação do dispositivo & disponível & em: \\
<https://wiki.wemos.cc/products:Iolin32:Iolin32>. & &
\end{tabular}

linguagem Arduino e a IDE de desenvolvimento Atom ${ }^{3}$ que é compatível com o ESP32. O software tem função de coordenar a ilha e captar os dados de tensão e corrente que passam pela ilha no momento em que algum aparelho conectado a ela estiver em funcionamento.

\subsection{Sistema}

Na Figura 1 segue a representação de um possível sistema integrado, usando a llha de medição de grandezas elétricas construída. A ilha envia essas informações para o microcomputador Raspberry $\mathrm{Pi}$, que por sua vez, repassa essas informações para o usuário, podendo usar um servidor intermediário como loT Cloud. Ainda na Figura 1, o usuário pode fazer o processo inverso, enviando dados de acionamento de algum dispositivo para a ilha de medição usando um microcomputador ou um servidor intermediário.

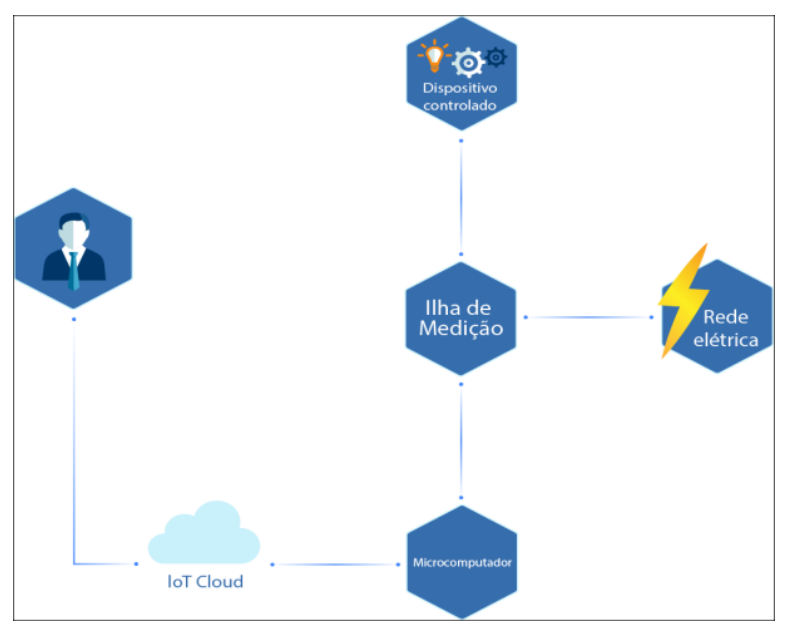

Figura 1: Diagramação de um sistema integrado.

\section{Desenvolvimento}

As informações de tensão e corrente são lidas e armazenadas em vetores. A ideia é que estes vetores correspondam à melhor resolução possível para os sinais de tensão e corrente, tanto no que se refere aos seus valores instantâneos, quanto o número de amostras dentro de um ciclo das ondas (frequência $60 \mathrm{hz}$, período $16,67 \mathrm{~ms}$ ).

Para determinar o tamanho exato dos vetores criados foi necessário realizar um teste com o processador do ESP32, usando a função micros( $)^{4}$ medindo-se quantos dados o microcontrolador é capaz de ler dos canais ADC durante $16,67 \mathrm{~ms}$. Após a medição, verificou-se que o ESP32 consegue ler 1667 valores por período de

\footnotetext{
3 Especificação do software disponível em: <https://atom.io/>.

Especificação da função e biblioteca disponível em: <https://www.arduino.cc/reference/pt/language/functions/time/micros/>.
} 
onda. Separando essa quantidade de valores em dois vetores, os vetores de tensão e corrente podem conter até 833 valores. Após realizar o laço de repetição novamente preenchendo-se os dois vetores de tamanho 833 com os valores lidos dos canais ADC, foi observado que a composição da memória interna onde ficam armazenadas as estruturas de dados não comportam um vetor com essa quantidade de valores. A solução foi a criação de uma variável fantasma (dummy) fazendo com que o período da onda corresponda a vetores com 556 valores, para tensão e para corrente.

A Figura 2 representa o processo de preenchimento dos vetores de tensão e corrente, fazendo a coleta das amostras necessárias para formar o período de onda no tempo certo.

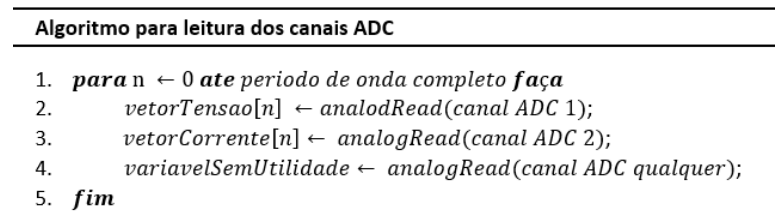

Figura 2: Pseudocódigo de leitura dos canais ADC e armazenamento nas estruturas.

Após ler os canais ADC e preencher os vetores, é preciso converter esses dados para que seja possível interpretá-los, isso por que o ESP32 converte o sinal no pino correspondente em valores entre $0 \sim 4095$. A equação 1 representa a conversão desses valores.

$$
\text { Valor }=\frac{(\text { Valor lido } *(\text { Voltagem de operação }))}{\text { Precisão canal ADC }}
$$

Onde:

- Valor lido = valor lido no canal ADC.

- Voltagem de operação = 3,3 Volts de acordo com a especificação do microcontrolador.

- Precisão do canal $A D C=12$ bits $=2^{12}=4.096$ $(0 \sim 4095)$.

Então, como mostra a Figura 3, basta usar o laço de repetição para percorrer os dois vetores novamente, mas agora, convertendo os valores lidos usando a equação 1.

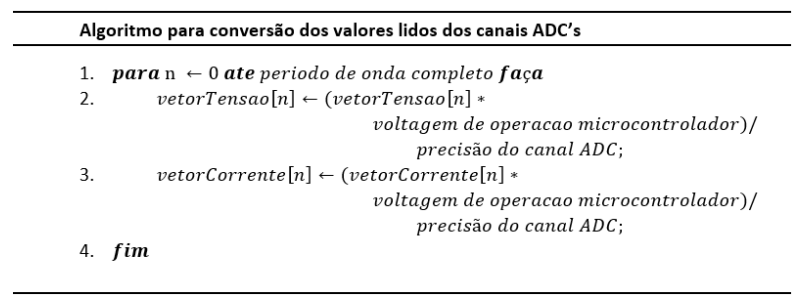

Figura 3: Pseudocódigo de conversão de valores lidos dos canais ADC.
Para que os valores lidos dos canais ADC sejam compatíveis com as grandezas reais é preciso adicionar constantes que são capazes de transformar os valores em que o circuito trabalha nos valores reais de tensão e corrente que o amperímetro é capaz de medir. A equação 2 representa o ajuste de valores para a tensão.

$$
\begin{aligned}
& \text { Valor }=(\text { Valor Lido }- \text { Voltagem de operacao }) * \\
& \text { Constante de Operação da Tensão }
\end{aligned}
$$

Onde:

- $\quad$ Valor lido $=$ Valor lido no canal ADC.

- Voltagem operação = 3,3 Volts / $2=1,65 \mathrm{~V}$.

- $\quad$ Constante de operação da tensão $=142.7$ V/V.

A correção a cima permite que uma grandeza alternada seja compatibilizada com a entrada do ADC além de compensar o ganho do circuito de condicionamento de sinais. De forma análoga, o mesmo ajuste é aplicado aos valores de corrente na equação 3 :

$$
\begin{gathered}
\text { Valor }=(\text { Valor lido }- \text { Voltagem de operacao }) * \\
\text { Constante de Operação da Corrente }
\end{gathered}
$$

Onde:

- $\quad$ Valor lido = Valor lido no canal ADC.

- Voltagem operação = 3,3 Volts / $2=1,65 \mathrm{~V}$.

- Constante de operação da corrente $=8 \mathrm{~A} / \mathrm{V}$.

A Figura 4 representa a aplicação das equações 2 e 3 nos vetores de tensão e corrente, os transformando assim, em vetores com valores reais da grandeza que vão possibilitar a realização dos cálculos de medição.

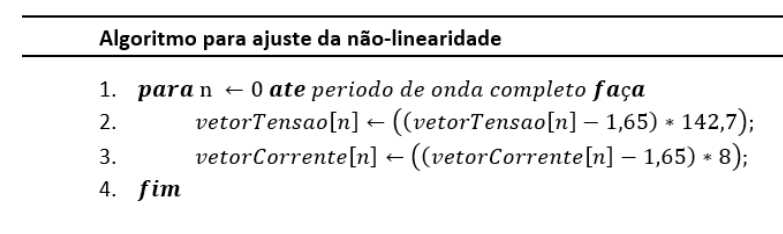

Figura 4: Pseudocódigo para conversão e ajuste de valores.

Segundo a equação 4, potência instantânea pode ser definida como a quantidade de energia gasta em um período de tempo infinitesimal.

$$
\text { Potência Instantânea }(t)=U(t) \cdot I(t)
$$

Onde $U(t)$ e $\mathrm{I}(t)$ são tensão e corrente respectivamente, em um dado instante $t$. Representado na Figura 5, um vetor auxiliar de potência instantânea é preenchido 
percorrendo-se os vetores de tensão e corrente aplicando a equação 4.

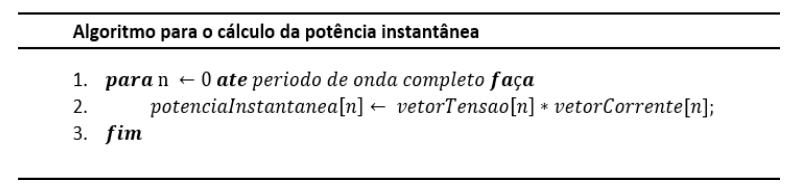

Figura 5: Pseudocódigo do cálculo de potência instantânea.

Com o vetor de potência instantânea preenchido, agora basta calcular a potência instantânea acumulada. Como mostra a Figura 6 , a potência instantânea acumulada representa a soma de todos os valores de potência instantânea calculados na Figura 5.

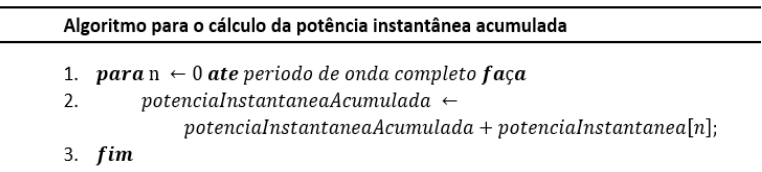

Figura 6: Pseudocódigo do cálculo da potência instantânea acumulada.

A potência média de um dispositivo conectado a placa é dada pela equação 5 .

$$
\text { Potência Média }=\frac{1}{n} \sum_{i=1}^{n} P i
$$

Onde $n$ representa a quantidade de amostras ou tamanho do vetor. Visto que a potência instantânea acumulada já foi calculada, basta aplicar a equação 6 .

$$
\text { Potencia Média }=\frac{\text { Potencia Instantânea Acomulada }}{\text { Tamanho do Vetor }}
$$

O valor eficaz pode ser calculado usando a equação 7 .

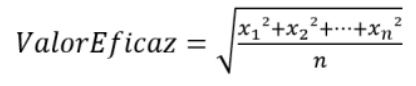

Onde $x$ é o valor instantâneo de tensão ou corrente e $n$ representa o número de amostras ao longo do período de onda completo. Como pode ser observado na Figura 7, para calcular o valor eficaz de tensão, basta realizar a soma do quadrado dos valores do vetor de tensão em uma variável auxiliar, dividir a soma desses valores pelo período de onda completo e então calcular a raiz quadrada deste valor.

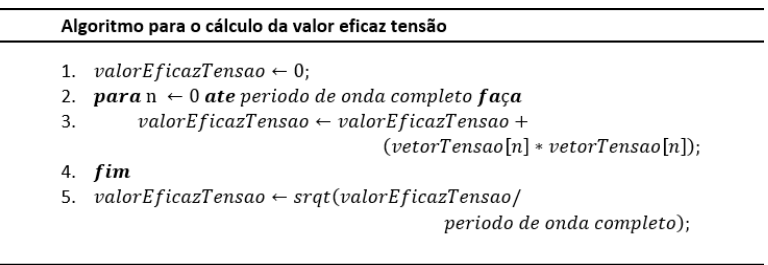

Figura 7: Pseudocódigo do cálculo do valor eficaz da tensão.
Tem-se então o valor eficaz da tensão calculado e armazenado na respectiva variável. O processo representado na Figura 8 é análogo ao processo da Figura 7, porém, com a nova variável auxiliar representando $o$ valor eficaz da corrente e consequentemente usando 0 vetor de corrente representando os valores a serem somados.

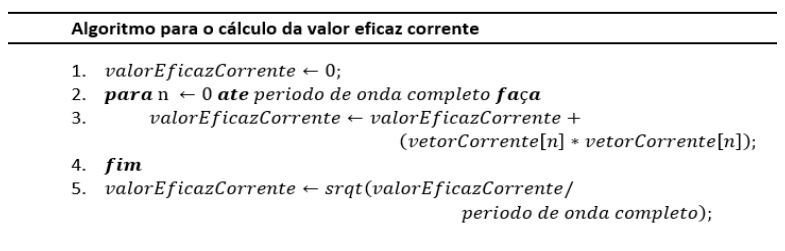

Figura 8: Pseudocódigo do cálculo do valor eficaz da corrente.

Com os valores eficazes de tensão e corrente calculados, agora finalmente é possível calcular o fator de potência. $O$ fator de potência é calculado a partir da equação 8.

$$
\text { Fator de Potência }=\frac{\text { Potência Média }}{\text { Valor Eficaz de Tensăo }+ \text { Valor Ef ficaz Corrente }}
$$

Os valores de potência média, valor eficaz de tensão, valor eficaz de corrente e fator de potência calculados, são informações apresentáveis ao usuário. Calcula-se uma média móvel dos valores antes da apresentação final.

\section{Resultados}

Apresentada na Figura 9, a ilha de medição de grandezas elétricas contém 0 circuito de condicionamento de sinais, um ESP32 e o relé, além da fonte de alimentação.

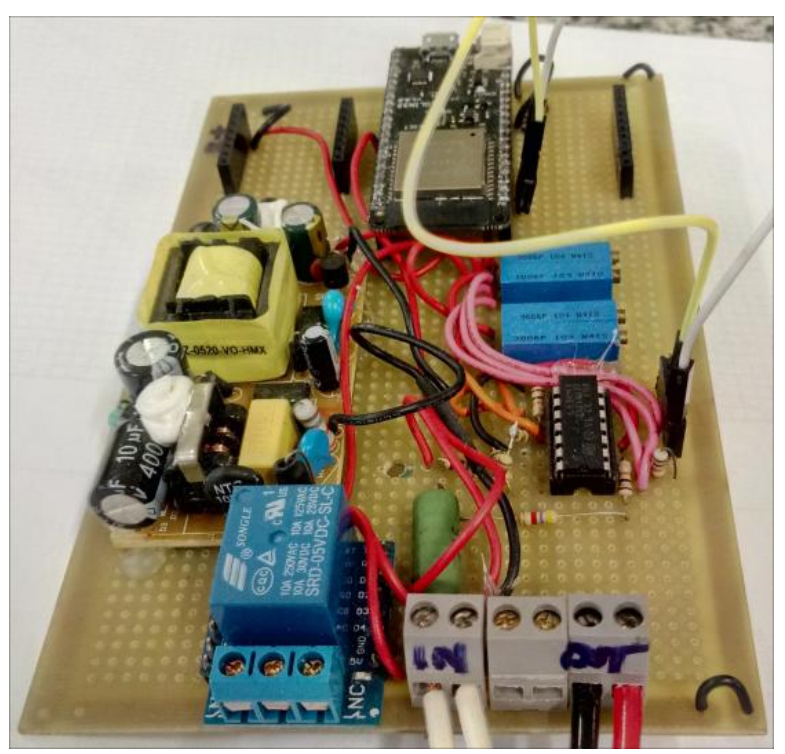

Figura 9: Ilha de medição de grandezas elétricas. 
O sistema desenvolvido apresentado na Figura 10 é classificado como um sistema integrado, ou seja, um sistema que consegue operar utilizando uma central inteligente. Trata-se de um sistema de automação simples, baseado em uma ilha de medição que pode ser observada por um microcomputador e que é controlada por um microcontrolador Wemos Lolin ESP32.

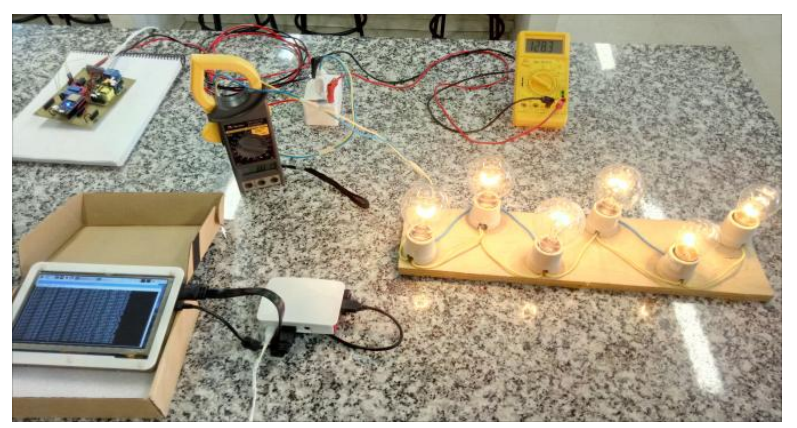

Figura 10: Sistema completo: ilha de medição, Raspberry Pi, carga elétrica, voltímetro e amperímetro.

$\mathrm{Na}$ figura 10 a ilha de monitoramento tem como dispositivo conectado a ela uma série de lâmpadas incandescentes ligadas em paralelo. Nas figuras $11 \mathrm{e}$ 12 é possível observar as informações geradas pelo sistema em um servidor Web local gerado no próprio microcontrolador.

\begin{tabular}{|l|l|}
\hline ESP32 Dados Servidor WEB $\times$ & + \\
$\leftarrow \rightarrow C$ C & (i) 192.168.43.51 \\
Informacoes Importantes: \\
Potencia Media: $145.53 \mathrm{~W}$ \\
Valor Eficaz da Tensao: $122.82 \mathrm{~V}$ \\
Valor Eficaz de Corrente: $1.21 \mathrm{~A}$ \\
Fator de Potencia: 0.98 \\
\hline
\end{tabular}

Figura 11: Apresentação dos dados de tensão e corrente eficazes, potência média e fator de potência para o conjunto de lâmpadas usado.

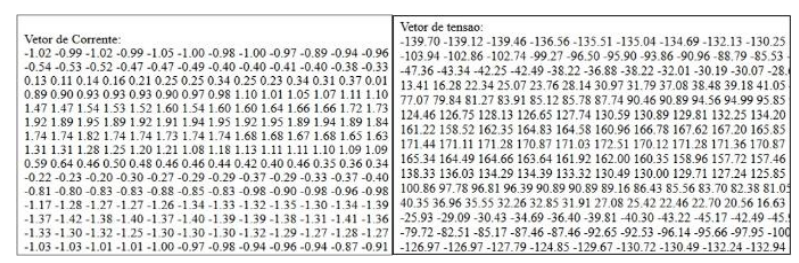

Figura 12: Amostragem dos dados que representam os vetores de corrente e tensão correspondente a um período de onda de $60 \mathrm{~Hz}$.

Após a primeira bateria de testes, foram gerados os gráficos off-line com os vetores de tensão e corrente lidos no servidor Web do ESP32. Foi observado, como mostra o Gráfico 1, que o canal ADC do ESP32 acaba gerando valores não-lineares. O fabricante do ESP32 Espressif divulgou no manual e documentação que os chips do ESP32 podem apresentar uma diferença de mais ou menos $6 \%$ de um chip para outro nos resultados medidos usando os canais ADC (EXPRESSIF SYSTEMS, 2018, p. 23). Além disso, a sua conversão não possui uma resposta linear por toda faixa disponível e sugere que os usuários implementem outros métodos além do citado no datasheet caso julguem necessários.

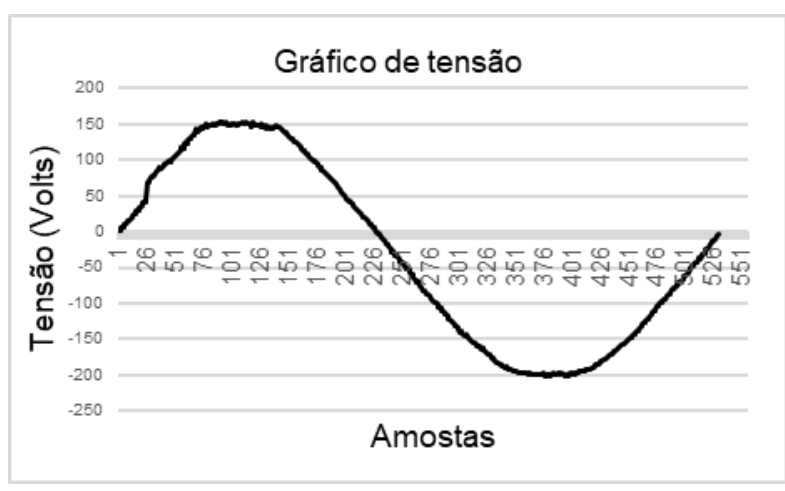

Gráfico 1: Achatamento da senoide de tensão devido à não-linearidade do canal ADC.

Para a correção da não linearidade foi preciso estudar o comportamento da função, o que foi conseguido com a aplicação de valores conhecidos de tensão no canal ADC. Optou-se por usar a ferramenta Microsoft Excel ${ }^{5}$ para gerar o Gráfico 2 encontrando o valor de $\mathrm{R}^{2}$ (coeficiente de determinação) a partir de um polinômio de terceiro grau. A ferramenta retorna à equação que melhor representa o modelo.

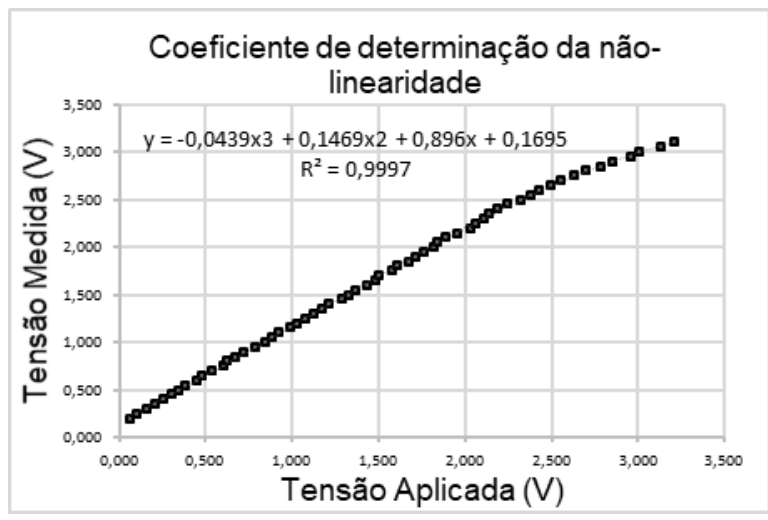

Gráfico 2: Equação e coeficiente de determinação relativos à não linearidade do canal ADC.

A equação gerada pelo software Microsoft Excel foi aplicada aos vetores de tensão e corrente para realizar a correção da não-linearidade do canal ADC como mostra a Figura 13.

\footnotetext{
5 Especificação do software disponível em: $<$ https://products.office.com/pt-br/excel>.
} 
Algoritmo para ajuste da não-linearidade

1. para $\mathrm{n} \leftarrow 0$ ate periodo de onda completo faça

vetorTensao $[n] \leftarrow(-0.0439 *$ (vetorTensao $[n] *$ vetorTensao $[n]$ vetorTensao $[n]))+(0.1469 *($ vetorTensao $[n] *$ vetorTensao $[n]))+(0.896 *$ vetorTensao $[n])+0.1695$

3. vetorCorrente $[n] \leftarrow(-0.0439 *$ (vetorCorrente $[n]$ * vetorCorrente $[n] *$ vetorCorrente $[n]))+$ $(0.1469 *($ vetorCorrente $[n]$ * vetorCorrente $[n]))+(0.896 *$

4. fim vetorCorrente $[n])+0.1695$

Figura 13: Pseudocódigo para correção da não-linearidade.

Com a correção da não linearidade do canal ADC do ESP32, foi possível gerar os gráficos de tensão e corrente mais precisos como pode-se observar no Gráfico 3.

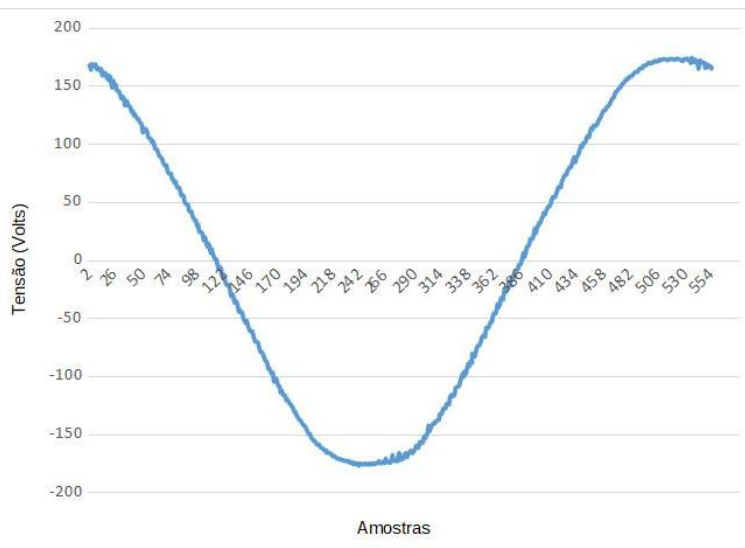

Gráfico 3: Gráfico de tensão após a correção da não linearidade

Os valores medidos pela ilha e disponibilizados através do servidor Web foram comparados com os valores medidos com um Wattímetro digital portátil marca Politerm, modelo POL64 ${ }^{6}$. A tensão na entrada variou entre 100 e 150 volts e a potência entre 0 e 200 watts. Os testes foram feitos considerando-se apenas cargas resistivas, ou seja, com fator de potência unitário, embora cargas com outros fatores de potência tenham sido testadas com resultados concordantes entre 0 wattímetro e a ilha.

Os gráficos a seguir indicam a relação entre os valores medidos com a ilha e os valores medidos com o wattímetro para cada uma das grandezas de interesse: valores eficazes de corrente e tensão, potência ativa e fator de potência. É importante frisar que estes testes foram realizados antes da calibração da ilha e que as constantes multiplicativas usadas haviam sido estimadas sem maior rigor pelo entendimento de que esta análise ajudaria na identificação acurada destas constantes.

\footnotetext{
6 Especificação do dispositivo disponível em: $<$ http://www.politerm.com.br/Produto-c-1-Wattimetro-Digital-modeloPOL-.aspx>
}

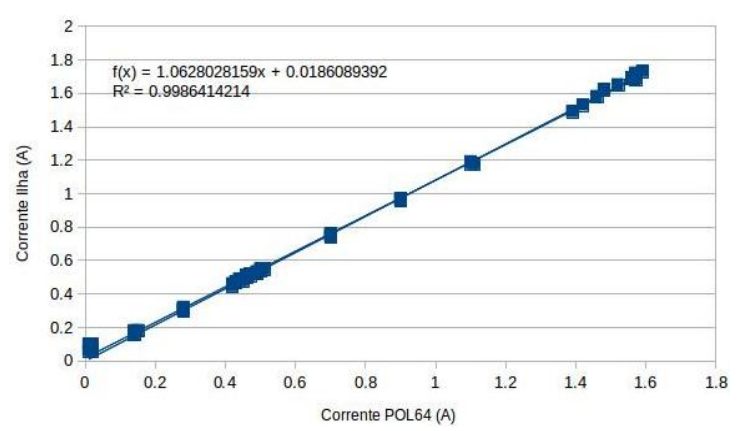

Gráfico 4: Valor eficaz de corrente.

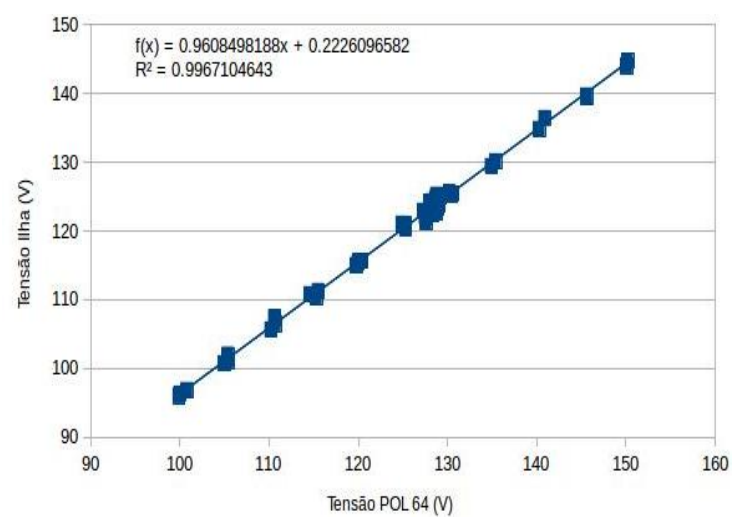

Gráfico 5: Valor eficaz de tensão.

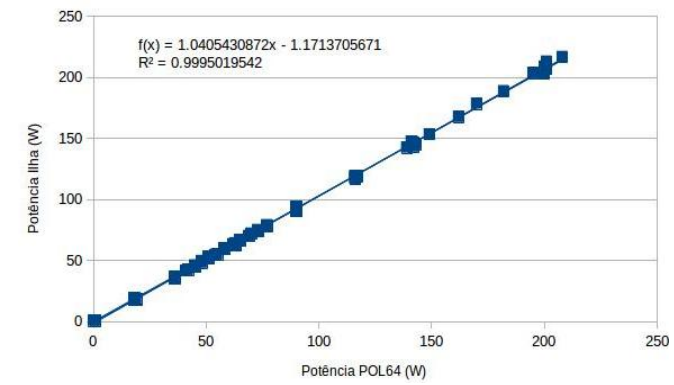

Gráfico 6: Potência ativa

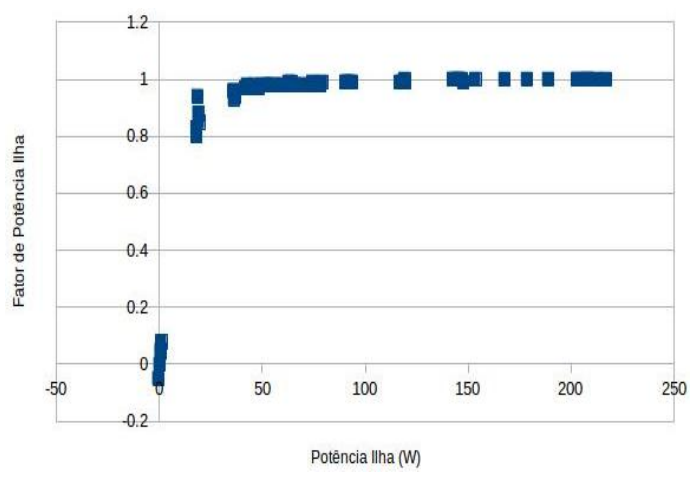

Gráfico 7: Fator de potência.

Os gráficos apresentam coeficientes angulares não unitários o que indica a necessidade de ajuste das constantes multiplicativas, que pode ser feito facilmente no software da ilha ou com a calibração do circuito 
condicionador de sinais. Por outro lado, a expectativa é que os coeficientes lineares sejam nulos, e o que se verifica é que os mesmos são pequenos, mas não nulos. Esta correção também pode ser facilmente feita com a calibração do off-set na entrada do conversor AD ou mesmo por software. O ideal é que haja um procedimento padronizado de calibração dos circuitos condicionadores de sinal para que o software das ilhas possa ser único e padronizado.

A calibração dos canais (corrente e tensão) também deve reduzir a incorreção dos valores de fator de potência para potência abaixo de 20 watts, como pode ser verificado no gráfico 5 .

\section{Conclusão}

O desenvolvimento deste projeto possibilitou a construção de um sistema integrado composto por um microcomputador e uma ilha de medição de grandezas elétricas. A ilha de medição foi capaz de medir grandezas elétricas e controlar um dispositivo conectado a ela. A manutenção das variáveis de apoio e calibrações de dados deve ser feita com testes exaustivos em ambientes diferentes e com equipamentos de medições diferentes.

O sistema produzido neste trabalho pode ser expandido, aumentando suas funcionalidades de tal forma que um microcomputador passe a controlar não só uma, mas várias ilhas de medição simultaneamente, que por sua vez poderão ter a capacidade de controlar vários dispositivos simultaneamente. Um produto com potencial de mercado pode advir da continuação e aperfeiçoamento do trabalho desenvolvido neste projeto.

Para realizar a comunicação entre o microcontrolador e o microcomputador, o protocolo MQTT se mostra capaz de atender a expectativas devido a sua topologia Publish/Subscribe, versatilidade e compatibilidade com a maioria dos microcomputadores e microcontroladores disponíveis no mercado. Além do aumento significativo na quantidade de ilhas sendo monitoradas por microcomputadores, as informações geradas pela ilha de monitoramento e as informações de acionamento podem ser enviadas e recebidas via servidor loT Cloud (do inglês: Nuvem loT), que é uma plataforma online projetada para armazenar e processar dados de Internet das Coisas.

Futuras versões da ilha de medição de grandezas elétricas poderão realizar mais processamento local (edge computing) e empregar outros microcontroladores alternativos e mais recentes com ADC de melhor qualidade, porém, de custo mais alto, inclusive com MicroPython (Python para microcontroladores) o que facilita o desenvolvimento em Internet das Coisas.

\section{Referências}

[1] MATTENRN, F.; FLOERKEMEIER, C. From the Internet of Computers to the Internet of Things. In: Lecture Notes in Computer Science. [S.I.: s.n., 2010].

[2] WEISER, Mark. Ubiquitous Computing. Computer, HOT TOPICS, IEEE Computer Society. [S.I.], 1993.

[3] WEISER, Mark. The computer for the 21st century. SIGMOBILE Mob. Comput. Commun. [S.I.], 1999.

[4] AQUINO, A. INTERNET DAS COISAS: Nós, as cidades, os robôs, os carros: Tudo conectado!. Computação Brasil. Porto Alegre: Sociedade Brasileira de Computação, n. 29, p. 1-58, mar. 2015.

[5] EGHAM, U.K. Gartner Says 8.4 Billion Connected "Things" Will Be in Use in 2017, Up 31 Percent From 2016. Gartner, 2017. Disponível em:<https://www.gartner.com/newsroom/id/3598917>. Acesso em: 27 maio 2019.

[6] EXPRESSIF SYSTEMS. ESP32 Series: Datasheet. 2018. Disponível em: <https://www.espressif.com/sites/default/files/document ation/esp32_datasheet_en.pdf>. Acesso em: 27 maio 2019. 